PROCEEDINGS OF THE

AMERICAN MATHEMATICAL SOCIETY

Volume 127, Number 9, Pages 2797-2803

S 0002-9939(99)04839-X

Article electronically published on April 15, 1999

\title{
CUT-POINT SPACES
}

\author{
B. HONARI AND Y. BAHRAMPOUR
}

(Communicated by Alan Dow)

\begin{abstract}
The notion of a cut-point space is introduced as a connected topological space without any non-cut point. It is shown that a cut-point space is infinite. The non-cut point existence theorem is proved for general (not necessarily $T_{1}$ ) topological spaces to show that a cut-point space is non-compact. Also, the class of irreducible cut-point spaces is studied and it is shown that this class (up to homeomorphism) has exactly one member: the Khalimsky line.
\end{abstract}

\section{INTRODUCTION}

The real line $\mathbf{R}$ is a source of intuition in topology. Many other familiar topological spaces can be obtained from $\mathbf{R}$ by topological constructions. It has the following properties:

(a) it is connected but the removal of any one of its points leaves it disconnected;

(b) it is metrizable;

(c) its topology can be generated by a linear ordering.

Conversely, it can be proved that every topological space with the above properties is homeomorphic to $\mathbf{R}$. Conditions $(b)$ and $(c)$ are too strong. They impose structures on the topological space, so this characterization of $\mathbf{R}$ seems somehow extrinsic.

In this paper we study the topological spaces that satisfy condition $(a)$, and call them cut-point spaces. In section 2, a cut-point space is defined again formally and some examples are given. In section 3 , it is shown that every cut-point space has an infinite number of closed points. Also, it is proved that every cut-point space is non-compact. To prove the latter, we need the most general form of the non-cut point existence theorem. The special case of this theorem for metric topological spaces is proved in [4]. A proof of the theorem for $T_{1}$ topological spaces can be found in [1] (see also [5]). In Section 4, an irreducible cut-point space is defined naturally as a cut-point space whose proper subsets are not cut-point spaces. It is shown that an irreducible cut-point space is necessarily homeomorphic to the Khalimsky line (see Example 2.5 for the definition of the Khalimsky line). This result may also be viewed as a straightforward characterization of the Khalimsky line. Objects in $n$-dimensional digital images have sometimes been regarded as subspaces of the product of $n$ copies of the Khalimsky line [2], [3].

Received by the editors March 3, 1997 and, in revised form, November 20, 1997.

1991 Mathematics Subject Classification. Primary 54F15, 54F65.

Key words and phrases. Continuum, cut-point space, cut point, non-cut point existence theorem, irreducible cut-point space, Khalimsky line.

(C)1999 American Mathematical Society 
Remark. Let $X$ be a topological space and let $Y \subseteq X$. Everywhere in this paper, the topology of $Y$ is the subspace topology. A point $x \in X$ is said to be closed (resp. open) if $\{x\}$ is a closed (resp. open) subset of $X$.

\section{Definitions And examples}

2.1. Definition. Let $X$ be a nonempty connected topological space. A point $x$ in $X$ is said to be a cut point of $X$ if $X \backslash\{x\}$ is a disconnected subset of $X$. A nonempty connected topological space $X$ is said to be a cut-point space if every $x$ in $X$ is a cut point of $X$.

In the following three examples, $\mathbf{R}^{2}$ is the Euclidean plane with the standard topology.

2.2. Example. The union of $n$ straight lines in $\mathbf{R}^{2}$ is a cut-point space if and only if either all of them are concurrent or exactly $n-1$ of them are parallel.

2.3. Example. Let $X_{1}=\left\{(x, y) \in \mathbf{R}^{2}: x \leq 0\right.$ and $\left.|y|=1\right\}$ and let $X_{2}=\left\{(x, y) \in \mathbf{R}^{2}: x>0\right.$ and $\left.y=\sin \frac{1}{x}\right\}$. Define $X=X_{1} \cup X_{2}$. Then $X$ is a cut-point space. For each $x \in X, X \backslash\{x\}$ has exactly two components.

A "connected ordered topological space" (COTS) is a connected topological space $X$ with the following property: if $Y$ is a three-point subset of $X$, there is a $y$ in $Y$ such that $Y$ meets two connected components of $X \backslash\{y\}$ (see [2]). Put $Y=\{(0,-1),(1, \sin 1),(0,1)\}$ in Example 2.3 to see that $X$ is not a COTS.

2.4. Example. Let $X_{0}=\left\{(x, 0) \in \mathbf{R}^{2}: x \leq 0\right\} \cup\left\{(x, 1) \in \mathbf{R}^{2}: x>0\right\}$ and let for each positive integer $n, Y_{n}=\left\{\left(\frac{1}{n}, y\right) \in \mathbf{R}^{2}: 0<y \leq 1\right\}$. Define $X=X_{0} \cup\left(\bigcup_{n=1}^{\infty} Y_{n}\right)$. Then $X$ is a cut-point space.

A connected topological space is said to have the "connected intersection property" if the intersection of every two connected subsets of it is connected. In Example 2.4, let $X_{1}=X_{0} \cup\left(\bigcup_{n=1}^{\infty} Y_{2 n-1}\right)$ and $X_{2}=X_{0} \cup\left(\bigcup_{n=1}^{\infty} Y_{2 n}\right)$. Since $X_{1} \cap X_{2}=X_{0}$ is not connected, $X$ does not possess the connected intersection property. Example 2.4 is a slightly modified version of an example in [6].

2.5. Example (The Khalimsky line). Let $\mathbf{Z}$ be the set of integers and let

$$
\mathcal{B}=\{\{2 i-1,2 i, 2 i+1\}: i \in \mathbf{Z}\} \cup\{\{2 i+1\}: i \in \mathbf{Z}\} .
$$

Then $\mathcal{B}$ is a base for a topology on $\mathbf{Z}$. The set $\mathbf{Z}$ with this topology is a cutpoint space and is called the Khalimsky line. Each point in $\mathbf{Z}$ has a smallest open neighborhood and the base $\mathcal{B}$ is the collection of all such neighborhoods. It can be easily seen that the Khalimsky line is irreducible in the sense that no proper subset of it is a cut-point space.

\section{Topological PRoperties of CUT-POINT SPACES}

Theorem 3.2 is the key theorem of this section. The main theorem of this section is Theorem 3.9 which implies the non-compactness of cut-point spaces. Notation 3.1 is adopted from [5].

3.1. Notation. Let $Y$ be a topological space. We write $Y=A \mid B$ to mean $A$ and $B$ are two nonempty subsets of $Y$ such that $Y=A \cup B$ and $A \cap \bar{B}=\bar{A} \cap B=\varnothing$. 
3.2. Theorem. Let $X$ be a connected topological space, and let $x$ be a cut point of $X$ such that $X \backslash\{x\}=A \mid B$. Then $\{x\}$ is open or closed. If $\{x\}$ is open, then $A$ and $B$ are closed; if $\{x\}$ is closed, then $A$ and $B$ are open.

Proof. Since $A$ is both open and closed in $X \backslash\{x\}$, there is an open subset $V$ of $X$ such that $A=V \cap(X \backslash\{x\})=V \backslash\{x\}$, and there is a closed subset $F$ of $X$ such that $A=F \cap(X \backslash\{x\})=F \backslash\{x\}$. Thus $A=V \backslash\{x\}=F \backslash\{x\}$. Since the assumption $V=F$ contradicts the connectedness of $X$, we have $\{x\}=V \backslash F$ or $\{x\}=F \backslash V$. If $\{x\}=V \backslash F$, then $\{x\}$ is open and $A=F$ is closed. If $\{x\}=F \backslash V$, then $\{x\}$ is closed and $A=V$ is open.

3.3. Corollary. Let $X$ be a connected topological space, and let $Y$ be the subset of all cut points of $X$. Then the following statements are obviously true.

(a) Every nonempty connected subset of $Y$ that is not a singleton, contains at least one closed point.

(b) If $x \in Y$ is open, then every limit point of $\{x\}$ in $Y$ is a closed point.

3.4. Lemma. Let $X$ be a connected topological space, and let $x$ be a cut point of it. If $X \backslash\{x\}=A \mid B$, then $A \cup\{x\}$ is connected.

Proof. If $A \cup\{x\}$ is not connected, then there are subsets $C$ and $D$ of $X$ such that $A \cup\{x\}=C \mid D$. Without loss of generality, we may assume that $x \in C$. Then $D \subseteq A$. Since $\overline{(B \cup C)} \cap D=(\bar{B} \cap D) \cup(\bar{C} \cap D)=\bar{B} \cap D \subseteq \bar{B} \cap A=\varnothing$, $\overline{(B \cup C)} \cap D=\varnothing$. Since $(B \cup C) \cap \bar{D}=(B \cap \bar{D}) \cup(C \cap \bar{D})=(B \cap \bar{D}) \subseteq B \cap \bar{A}=\varnothing$, $(B \cup C) \cap \bar{D}=\varnothing$. Therefore $X=(B \cup C) \mid D$. This contradicts the connectedness of $X$.

3.5. Lemma. Let $X$ be a connected topological space and let $x$ be a cut point of it. If $X \backslash\{x\}=A \mid B$ and if every point of $A$ is a cut point of $X$, then $A$ contains at least one closed point.

Proof. Suppose that $A$ consists exclusively of open points. Since, by Lemma 3.4, $A \cup\{x\}$ is connected, $\{x\}$ is closed and hence (by Theorem 3.2) $A \cup\{x\}$ is closed too. Thus, for every $y \in A, \overline{\{y\}} \subseteq A \cup\{x\}$, and therefore, by Corollary $3.3(b), x$ is the only possible limit point of $\{y\}$. As $\{y\}$ has a limit point (since $\{y\}$ is open and $X$ is connected), $x$ is a limit point of $\{y\}$. This implies that $\{x, y\}$ is connected for any $y \in A$. Let $y_{0} \in A$. Since $B \cup\{x\}$ is connected by Lemma 3.4,

$$
X \backslash\left\{y_{0}\right\}=\bigcup_{y \in A, y \neq y_{0}}\{x, y\} \cup(B \cup\{x\})
$$

is connected too. This contradicts the fact that $y_{0}$ is a cut point of $X$.

3.6. Lemma. Let $X$ be a connected topological space, and let $x$ and $y$ be two cut points of it such that $X \backslash\{x\}=A \mid B$ and $X \backslash\{y\}=C \mid D$. If $x \in C$ and $y \in A$, then $D \subseteq A$ and $B \subseteq C$.

Proof. Since $D \cup\{y\}$ is connected by Lemma 3.4, and since $D \cup\{y\} \subseteq X \backslash\{x\}$, we have $D \cup\{y\} \subseteq A$ or $D \cup\{y\} \subseteq B$. Since $y \in A$, the second inclusion is not true. Hence $D \subseteq A$. A similar argument shows that $B \subseteq C$.

In the next theorem, we show that a finite topological space cannot be a cut-point space. 
3.7. Theorem. Let $X$ be a cut-point space. Then the set of closed points of $X$ is infinite.

Proof. By mathematical induction, we construct a sequence $x_{1}, x_{2}, \cdots$ of distinct closed points in $X$. Define $C_{0}=X$. By Corollary $3.3(a)$, there exists a closed point $x_{1}$ in $C_{0}$. Since $x_{1}$ is a cut point of $X$, there are open subsets $C_{1}$ and $D_{1}$ of $X$ such that $X \backslash\left\{x_{1}\right\}=C_{1} \mid D_{1}$. Now, suppose that the distinct closed points $x_{1}, x_{2}, \cdots, x_{n}$ in $X$ and the open subsets $C_{1}, \cdots, C_{n}, D_{1}, \cdots, D_{n}$ of $X$ are chosen such that $X \backslash\left\{x_{i}\right\}=C_{i} \mid D_{i}, x_{i} \in C_{i-1}$ and $C_{i-1} \supseteq C_{i}$ for each $i, 1 \leq i \leq n$. According to Lemma 3.5, there is a closed point $x_{n+1} \in C_{n}$. There are open subsets $C_{n+1}$ and $D_{n+1}$ of $X$ such that $X \backslash\left\{x_{n+1}\right\}=C_{n+1} \mid D_{n+1}$. By interchanging $C_{n+1}$ and $D_{n+1}$, if necessary, we may asuume that $x_{n} \in D_{n+1}$. Thus, by Lemma 3.6, $C_{n} \supseteq C_{n+1}$. Since $x_{i} \notin C_{i}, x_{i} \notin C_{n}$ for any $i, 1 \leq i \leq n$. The fact that $x_{n+1} \in C_{n}$ implies that $x_{n+1}$ is different from $x_{1}, \cdots, x_{n}$.

3.8. Corollary. Let $X$ be a cut-point space. Then $|X|=\infty$.

Of course, Theorem 3.7 is a generalization of Corollary 3.8. Using the Hausdorff Maximal Principle, we prove another generalization of Corollary 3.8 in the following theorem.

3.9. Theorem. Let $X$ be a compact connected topological space with more than one point. Then $X$ has at least two non-cut points.

Proof. Suppose that $X$ has at most one non-cut point. Let $x_{0}$ be a cut point of $X$ and let $X \backslash\left\{x_{0}\right\}=A_{0} \mid B_{0}$. Since $X$ has at most one non-cut point, either $A_{0}$ or $B_{0}$ (without loss of generality assume $A_{0}$ ) exclusively consists of cut points. By Lemma 3.5, $A_{0}$ contains some closed cut point of $X$, say $x$. Let $X \backslash\{x\}=A \mid B$ and without loss of generality assume that $x_{0} \in B$. Then by Lemma 3.6, $A \subseteq A_{0}$. Define $\mathcal{S}=\{U: U$ is an open subset of $X, U \supseteq B, \bar{U} \backslash U$ is a singleton, and $\bar{U} \neq X\}$. Since $B$ is open and $\bar{B}=B \cup\{x\}, B \in \mathcal{S}$. For each $U_{\alpha} \in \mathcal{S}$ and $U_{\beta} \in \mathcal{S}$, write $U_{\alpha} \leq U_{\beta}$ if $U_{\alpha}=U_{\beta}$, or if $\bar{U}_{\alpha} \subseteq U_{\beta}$. $(\mathcal{S}, \leq)$ is clearly a partially ordered set, and by the Hausdorff Maximal Principle there is a maximal chain $\mathcal{C}$ in $\mathcal{S}$. Let $U_{\alpha} \in \mathcal{S}$, and let $\left\{x_{\alpha}\right\}=\bar{U}_{\alpha} \backslash U_{\alpha}$. Since $X \backslash\left\{x_{\alpha}\right\}=U_{\alpha} \mid\left(X \backslash \bar{U}_{\alpha}\right)$, by Lemma 3.5 there is a closed point $y \in X \backslash \bar{U}_{\alpha} \subseteq A$. Let $X \backslash\{y\}=C \mid D$. Since $\bar{U}_{\alpha}$ is connected by Lemma 3.4, $\bar{U}_{\alpha} \subseteq C$ or $\bar{U}_{\alpha} \subseteq D$, i.e. $U_{\alpha}<C$ or $U_{\alpha}<D$. Since $U_{\alpha}$ was arbitrary in $\mathcal{S}, \mathcal{S}$ (and consequently $\mathcal{C}$ ) does not have a maximal element. Thus $\bigcup_{U \in \mathcal{C}} U=\bigcup_{U \in \mathcal{C}} \bar{U}$. Write $V=\bigcup_{U \in \mathcal{C}} U$. Since $\bar{U}$ is connected for each $U \in \mathcal{S}, V$ is connected too. We claim that $V=X$. Suppose otherwise. Then $X \backslash V$ is a nonempty closed subset of $X$. Since $X \backslash V \subseteq A$, every point in $X \backslash V$ is a cut point of $X$ and is either open or closed by Theorem 3.2. As $X \backslash V$ is not open (it is closed and $X$ is connected), the points of $X \backslash V$ cannot all be open, and so there is a closed cut point $x^{\prime}$ in $X \backslash V$. Let $X \backslash\left\{x^{\prime}\right\}=G \mid H$. Since $V$ is connected, $V \subseteq G$ or $V \subseteq H$. Assume (without loss of generality) that $V \subseteq G$. Since $G \in \mathcal{S}, U \leq G$ for any $U \in \mathcal{C}$. Since $\mathcal{C}$ does not have a maximal element, $G \notin \mathcal{C}$. This contradicts the maximality of the chain $\mathcal{C}$. Hence $V=X$, and therefore $\mathcal{C}$ is an infinite open covering of $X$. Since $\mathcal{C}$ is a chain without a maximal element, there is no finite subcovering of $\mathcal{C}$ for $X$. This contradicts the compactness of $X$.

3.10. Corollary. Let $X$ be a cut-point space. Then $X$ is non-compact. 


\section{IRREDUCIBLE CUT-POINT SPACES AND CHARACTERIZATION OF THE KHALIMSKY LINE}

In this section, we define an irreducible cut-point space and we show that it is necessarily homeomorphic to the Khalimsky line (see Example 2.5).

4.1. Definition. A cut-point space is said to be an irreducible cut-point space if no proper subset of it (with the subspace topology) is a cut-point space.

4.2. Lemma. Let $X$ be a cut-point space, let $x \in X$, and let $X \backslash\{x\}=A \mid B$. If $A$ is not connected, then $A \cup\{x\}$ is a cut-point space.

Proof. Put $Y=A \cup\{x\}$. Clearly $x$ is a cut point of $Y$. Let $y$ be an arbitrary point in $A$. Since $X \backslash\{y\}=(Y \backslash\{y\}) \cup(B \cup\{x\})$ is not connected, and since $x \in(Y \backslash\{y\}) \cap(B \cup\{x\})$, either $Y \backslash\{y\}$ or $B \cup\{x\}$ is disconnected. By Lemma 3.4, $B \cup\{x\}$ is connected. Thus $Y \backslash\{y\}$ is disconnected.

4.3. Corollary. If $X$ is an irreducible cut-point space, then, for every $x \in X$, $X \backslash\{x\}$ has exactly two components.

Proof. Let $X \backslash\{x\}=A \mid B$. Since $X$ is irreducible, $A \cup\{x\}$ and $B \cup\{x\}$ are not cut-point spaces. Thus, by Lemma $4.2, A$ and $B$ are connected.

4.4. Lemma. Let $X$ be an irreducible cut-point space, let $x \in X$ and let $X \backslash\{x\}=$ $A \mid B$. Then there are exactly two points $y \in A$ and $z \in B$ such that $\{x, y\}$ and $\{x, z\}$ are connected. Furthermore if $x$ is closed then $y$ and $z$ are open, and if $x$ is open then $y$ and $z$ are closed.

Proof. Since, by Corollary 4.3, $A$ is connected and since $X$ is an irreducible cutpoint space, $A$ has a non-cut point $y$; i.e. $A \backslash\{y\}$ is connected. We claim that $y$ is the unique point in $A$ such that $\{x, y\}$ is connected. First we prove that if $\left\{x, y^{\prime}\right\}$ is connected for some $y^{\prime} \in A$, then $y^{\prime}=y$. Let $y^{\prime}$ be a point in $A$ such that $\left\{x, y^{\prime}\right\}$ is connected. Suppose that $y^{\prime} \neq y$. Since, by Lemma 3.4, $B \cup\{x\}$ is connected, and since $X \backslash\{y\}=(A \backslash\{y\}) \cup(B \cup\{x\})$, the connectedness of $\left\{x, y^{\prime}\right\}$ implies the connectedness of $X \backslash\{y\}$ (a contradiction). To prove that $\{x, y\}$ is connected we consider two cases.

(1) $x$ is closed. In this case, $A$ is (open and) not closed but $A \cup\{x\}$ is closed (both by Theorem 3.2). Thus $x$ is a limit point of $A$. On the other hand, since $X \backslash\{y\}=(A \backslash\{y\}) \cup(B \cup\{x\})$ is not connected, $x$ is not a limit point of $A \backslash\{y\}$. Hence $x$ is a limit point of $\{y\}$.

(2) $x$ is open. In this case, $A$ is (closed and) not open but $A \cup\{x\}$ is open (both by Theorem 3.2). Thus there is a point $y^{\prime}$ in $A$ which is not an interior point of $A$. Since $y^{\prime}$ is an interior point of $A \cup\{x\}, y^{\prime}$ is a limit point of $\{x\}$. Hence $\left\{x, y^{\prime}\right\}$ is connected. Since, as we proved above, $y^{\prime}=y,\{x, y\}$ is connected.

A similar argument shows that there is a unique point $z$ in $B$ such that $\{x, z\}$ is connected. The last statement of the lemma is implied by Theorem 3.2 and the connectedness of $\{x, y\}$ and $\{x, z\}$.

4.5. Theorem. A topological space $X$ is an irreducible cut-point space if and only if $X$ is homeomorphic to the "Khalimsky line".

Proof. It can be easily seen that the Khalimsky line is an irreducible cut-point space. Let $X$ be an irreducible cut-point space. By mathematical induction, we find a subset $Y$ of $X$ that is homeomorphic to the Khalimsky line, and then, by 
irreducibility of $X$, we conclude that $X=Y$. Let $x_{0}$ be a closed point in $X$, and let $X \backslash\left\{x_{0}\right\}=A_{0} \mid B_{0}$. By Lemma 4.4 there are points $x_{-1}$ in $A_{0}$ and $x_{1}$ in $B_{0}$ such that $\left\{x_{-1}, x_{0}\right\}$ and $\left\{x_{0}, x_{1}\right\}$ are connected. Define $Y_{1}=\left\{x_{-1}, x_{0}, x_{1}\right\}$. Let $A_{1}$ be the component of $X \backslash\left\{x_{1}\right\}$ that contains $x_{0}$ and let $B_{1}$ be the other component of $X \backslash\left\{x_{1}\right\}$. Let $B_{-1}$ be the component of $X \backslash\left\{x_{-1}\right\}$ that contains $x_{0}$ and let $A_{-1}$ be the other component of $X \backslash\left\{x_{-1}\right\}$. Assume that for an arbitrary positive integer $n$, the subset $Y_{n}=\left\{x_{i}: i \in \mathbf{Z}\right.$ and $\left.-n \leq i \leq n\right\}$ of $X$ (with $2 n+1$ points) is chosen such that for each $i$ and $j$ which satisfy $-n \leq i, j \leq n$ and $|i-j|=1$, $\left\{x_{i}, x_{j}\right\}$ is connected. Moreover, assume that for each nonzero $i,-n \leq i \leq n$, the components $A_{i}$ and $B_{i}$ of $X \backslash\left\{x_{i}\right\}$ are chosen such that $x_{0} \in A_{i}$ if $i$ is positive, and $x_{0} \in B_{i}$ if $i$ is negative. Since $\left.Y_{n} \backslash\left\{x_{-n}\right\}=\bigcup x_{i}, x_{j}\right\}$ is connected, $-n<i<j \leq n, j=i+1$

it is a subset of $A_{-n}$ or $B_{-n}$, and since $x_{0} \notin A_{-n}, Y_{n} \backslash\left\{x_{-n}\right\} \subseteq B_{-n}$. By Lemma 4.4 there is a unique point $x_{-n-1}$ in $A_{-n}$ such that $\left\{x_{-n-1}, x_{-n}\right\}$ is connected. Since $\left(Y_{n} \cup\left\{x_{-n-1}\right\}\right) \backslash\left\{x_{n}\right\}=\bigcup_{-n-1<i<j<n, j=i+1}\left\{x_{i}, x_{j}\right\}$ is connected, it is a subset of $A_{n}$ or $B_{n}$, and since $x_{0} \notin B_{n},\left(Y_{n} \cup\left\{x_{-n-1}\right\}\right) \backslash\left\{x_{n}\right\} \subseteq A_{n}$. By Lemma 4.4 there is a unique point $x_{n+1}$ in $B_{n}$ such that $\left\{x_{n}, x_{n+1}\right\}$ is connected. Thus we obtain a subset $Y_{n+1}=\left\{x_{i}: i \in \mathbf{Z}\right.$ and $\left.-(n+1) \leq i \leq n+1\right\}$ of $X$ (with $2 n+3$ points) such that for each $i$ and $j$ which satisfy $-(n+1) \leq i, j \leq n+1$ and $|i-j|=1,\left\{x_{i}, x_{j}\right\}$ is connected. To complete the induction step, we define the subsets $A_{-n-1}, B_{-n-1}, A_{n+1}, B_{n+1}$ of $X$ such that $X \backslash\left\{x_{-n-1}\right\}=A_{-n-1} \mid B_{-n-1}$, $X \backslash\left\{x_{n+1}\right\}=A_{n+1} \mid B_{n+1}, x_{0} \in B_{-n-1}$ and $x_{0} \in A_{n+1}$. Put

$$
Y=\bigcup_{n=1}^{\infty} Y_{n}=\left\{x_{i}: i \in \mathbf{Z}\right\}
$$

It can be easily seen that for each integer $i, Y \cap A_{i}=\left\{x_{j}: j<i\right\}$ and $Y \cap B_{i}=$ $\left\{x_{j}: j>i\right\}$. Since $x_{0}$ is closed, (by iterated application of Lemma 4.4) $x_{n}$ is closed if $n$ is even, and $x_{n}$ is open if $n$ is odd. Clearly, for each $i \in \mathbf{Z}$, the smallest open neighborhood of $x_{2 i+1}$ in $Y$ is $\left\{x_{2 i+1}\right\}$. Since for each $i \in \mathbf{Z}, x_{2 i}$ is a limit point of $\left\{x_{2 i-1}\right\}$ and $\left\{x_{2 i+1}\right\}$, every open neighborhood of $x_{2 i}$ in $X$ (and hence in $Y$ ) contains $x_{2 i-1}$ and $x_{2 i+1}$. On the other hand, since $x_{2 i-2}$ and $x_{2 i+2}$ are closed, $B_{2 i-2}$ and $A_{2 i+2}$ are open in $X$. Thus $\left\{x_{2 i-1}, x_{2 i}, x_{2 i+1}\right\}=\left(Y \cap B_{2 i-2}\right) \cap\left(Y \cap A_{2 i+2}\right)$ is the smallest open neighborhood of $x_{2 i}$ in $Y$. Hence

$$
\mathcal{B}^{\prime}=\left\{\left\{x_{2 i-1}, x_{2 i}, x_{2 i+1}\right\}: i \in \mathbf{Z}\right\} \cup\left\{\left\{x_{2 i+1}\right\}: i \in \mathbf{Z}\right\}
$$

is a base for the topology of $Y$. Comparing this base with the base of the Khalimsky line in Example 2.5, we see that $Y$ is homeomorphic to the Khalimsky line.

\section{REFERENCES}

[1] J. G. Hocking, G.S. Young, Topology, Addison-Wesley (1961). MR 23:A2857

[2] E.D. Khalimsky, R. Kopperman, P.R. Meyer, "Computer graphics and connected topologies on finite ordered sets", Topology and its Applications, 36 (1990) 1-17. MR 92c:54037

[3] R. Kopperman, "The Khalimsky line in digital topology", in Y.-L. O et al., eds., Shape in Picture: Mathematical Description of Shape in Grey-Level Images, Springer-Verlag (1994) 3-20.

[4] R. L. Moore, "Concerning simple continuous curves", Trans. Amer. Math. Soc., 21 (1920) 333-347. 
[5] S. B. Nadler, Jr., Continuum Theory: An Introduction, Marcel Dekker (1992). MR 93m:54002

[6] G.T. Whyburn, "Cut points in general topological spaces", Proc. Nat. Acad. Sci., USA, 61 (1968) 380-387. MR 39:3463

Faculty of Mathematics, Shahid Bahonar University of Kerman, Kerman, Iran

E-mail address: honari@arg3.uk.ac.ir

E-mail address: bahram@arg3.uk.ac.ir 